\title{
Unstable Angina
}

National Cancer Institute

\section{Source}

National Cancer Institute. Unstable Angina. NCI Thesaurus. Code C66911.

Ang ina pectoris (or equivalent type of ischemic discomfort) which has recently changed in frequency, duration, intensity, or occurs at rest. 